\title{
Interrogation of Chromosome 13q12-14 in Esophageal Squamous Cell Carcinoma
}

\author{
Heidi S. Erickson ${ }^{1,2}$, Jaime Rodriguez-Canales ${ }^{1}$, Paul S. Albert ${ }^{3}$, Kris Yala ${ }^{4}$, Sumana Mukherjee ${ }^{1}$, \\ Nan $\mathrm{Hu}^{5}$, Alisa M. Goldstein ${ }^{5}$, Rodrigo F. Chuaqui ${ }^{1}$, Stephen A. Hewitt ${ }^{4}$, Philip R. Taylor ${ }^{5}$ and \\ Michael R. Emmert-Buck ${ }^{*}, 1$
}

\author{
${ }^{1}$ Pathogenetics Unit, Laboratory of Pathology, National Cancer Institute, National Institutes of Health, Bethesda, \\ Maryland 20892, USA \\ ${ }^{2}$ Thoracic/Head \& Neck Medical Oncology, Division of Cancer Medicine, University of Texas M.D. Anderson Cancer \\ Center, Houston, Texas 77030, USA \\ ${ }^{3}$ Biometric Research Branch, Division of Cancer Treatment and Diagnosis, National Cancer Institute, National \\ Institutes of Health, Bethesda, Maryland 20852, USA \\ ${ }^{4}$ Tissue Array Research Program, Laboratory of Pathology, National Cancer Institute, National Institutes of Health, \\ Bethesda, Maryland 20892, USA \\ ${ }^{5}$ Genetic Epidemiology Branch, Division of Cancer Epidemiology and Genetics, National Cancer Institute, National \\ Institutes of Health, Bethesda, Maryland 20892, USA
}

\begin{abstract}
Previous studies of esophageal squamous cell carcinoma (ESCC) suggested chromosome region 13q12-14 harbors a familial ESCC gene. DNA sequencing of the BRCA2 gene, located on 13q12, showed evidence of both germline and tumor specific alterations but the frequency of changes was low and did not fit the classic Knudsen two-hit gene inactivation model. To further investigate chromosome 13q12-14 in ESCC, quantitative expression measurements were performed on BRCA2 and 11 neighboring genes in matched normal epithelium and tumor from 17 cases. Transcript analysis showed normal levels of five genes, tumor down-regulation of two genes (TNFRS19 and TPT1), and tumor upregulation of five genes, including $B R C A 2$. No evidence of $B R C A 2$ loss-of-function was detected based on reduced mRNA in tumor cells. Between 13q12.3 (KATNAL1) and 13q12.3-q13 (CCNA1) five adjacent genes showed increased mRNA expression raising the possibility of a DNA amplicon; however, qPCR analysis showed normal DNA amounts in this region. CCNA1 transcript was significantly up-regulated in tumors and was thus further interrogated at the protein level by immunohistochemistry. CCNA1 staining was restricted to normal basal epithelium and was not expressed in more superficial, differentiated regions. In contrast, the CCNA1 protein was ubiquitously and highly expressed throughout tumor foci. Overall, these data from a relatively small number of cases (17) suggest that TNFRS19 and TPT1 deserve further investigation as candidate tumor suppressor genes in esophageal cancer in a larger patient series; BRCA2 mRNA is increased in the tumors, likely as a compensatory response to the marked DNA damage that is present in these lesions; and, CCNA1 was identified as a novel up-regulated gene in ESCC.
\end{abstract}

Keywords: Esophagus, carcinoma, squamous cell, chromosome 13q, BRCA2, genomic region, gene expression, upregulated region, microdissected tissue.

\section{INTRODUCTION}

Esophageal squamous cell carcinoma (ESCC) is the fifth leading cause of cancer death in the world, with a significantly higher incidence in certain geographic areas such as Shanxi Province in China. Epidemiological studies suggest that both environment and genetics influence ESCC development and there is evidence for Mendelian inheritance of a tumor suppressor gene (TSG) [1,2]. Genomic studies of ESCC have identified several chromosomal regions with high levels of loss of heterozygosity (LOH), including

*Address correspondence to this author at the Pathogenetics Unit, Laboratory of Pathology, National Cancer Institute, National Institutes of Health, Bethesda, Maryland 20892, USA; Tel: (301) 496-2912; Fax: (301) 5947582; E-mail: mbuck@helix.nih.gov chromosome 13 [1-3]. Additionally, a statistically significant difference in allelic loss on chromosome 13 was observed between sporadic versus familial cases suggesting this chromosome may harbor an inherited TSG $[4,5]$.

ESCC shows extensive chromosomal instability thus it has not been possible to precisely map a minimal deletion interval; however, both the tumor LOH data and the results comparing allelic loss in sporadic versus familial cases point to the genomic region spanning chromosome 13q12-14 as the most likely location of a putative esophageal cancer gene [6-11]. Direct DNA sequencing of selected candidates to date has not identified a classically inactivated TSG on chromosome 13, although the data on BRCA2 are difficult to interpret as missense mutations have been identified in over $10 \%$ of tumors, and in the germline of $5 \%$ of patients with a family history of ESCC [12-14]. Moreover, similar results of 
$B R C A 2$ germline mutations have been observed in high-risk ESCC populations in both Iran and India [15, 16].

One strategy to assess the status of candidate TSGs in a genomic interval of interest is to evaluate transcript levels. Alterations in mRNA in tumors can occur due to several different mechanisms, including homozygous DNA deletion, aberrant epigenetic regulation, decreased dosage due to haploinsufficiency, or decreased stability of mutated mRNAs [17]. In the present study we performed quantitative RTPCR (qRT-PCR) analysis on 12 genes on 13q12-14, including $B R C A 2$, to assess if any of the candidates exhibited loss-of-function based on reduced expression of mRNA. The gene set was selected to include genomic locations across the region of interest on chromosome 13, and genes with potential growth related functions.

\section{MATERIALS AND METHODS}

\section{Clinical Tissue Specimens}

Twenty-four cases comprising 52 frozen tissue blocks of newly diagnosed and untreated human esophageal squamous cell carcinoma (ESCC) were obtained from patients who underwent esophageal resection at Shanxi Cancer Hospital and Institute, Taiyuan in Shanxi Province of China. The specimens were collected under an IRB-approved protocol and transferred to the National Cancer Institute (NCI). The tissues were evaluated by a pathologist (JRC) and blocks from seventeen cases were selected for study based upon both histologic criteria (presence of ESCC epithelium and matched normal squamous epithelium) and microdissection criteria ( $\geq 10,000$ normal epithelial or tumor cells).

\section{Tissue Microdissection}

Matched normal epithelium and tumor were dissected from histological sections using laser capture microdissection (LCM) as described previously [18]. Frozen tissue samples were hematoxylin and eosin (H\&E) stained prior to LCM and approximately 10,000 cells were procured from each block (equivalent to $\sim 3,000$ LCM shots per tissue type), immediately placed into lysis buffer, and stored at $80^{\circ} \mathrm{C}$ until RNA extraction. A replicate microdissection of all samples was obtained for a second qRT-PCR analysis of $B R C A 2$ to assess reproducibility. Additional frozen tissue sections were available in five of seventeen cases (case numbers 9, 10, 11, 12, and 16) and were manually microdissected for subsequent DNA extraction.

\section{RNA Isolation, Quantitation, and Qualitation}

RNA extraction and isolation from samples were conducted as previously described $[19,20]$. Following RNA isolation, $3 \mu$ aliquots per sample were taken for RNA quantity and quality measurement and used immediately. All remaining RNA samples were stored at $-80^{\circ} \mathrm{C}$. Quantitation and qualitation of individual sample total RNA was conducted using NanoDrop (NanoDrop Technologies, Wilmington, DE, USA) and Bioanalyzer (Agilent Technologies, Inc., Santa Clara, CA, USA) equipment, respectively. Sample quality was assessed by RNA integrity number (RIN) using 2100 Expert software (Agilent Technologies, Inc.).

\section{Chromosome 13q Genes}

Twelve genes spanning 27.5 million bp between 13q11$13 q 14$ were selected for analysis (Fig. 1, Table 1). The genes were chosen such that they spanned the genomic region of interest on chromosome 13 and because reports in the literature suggested a potential role in cell growth $[3,8,21$, 22].

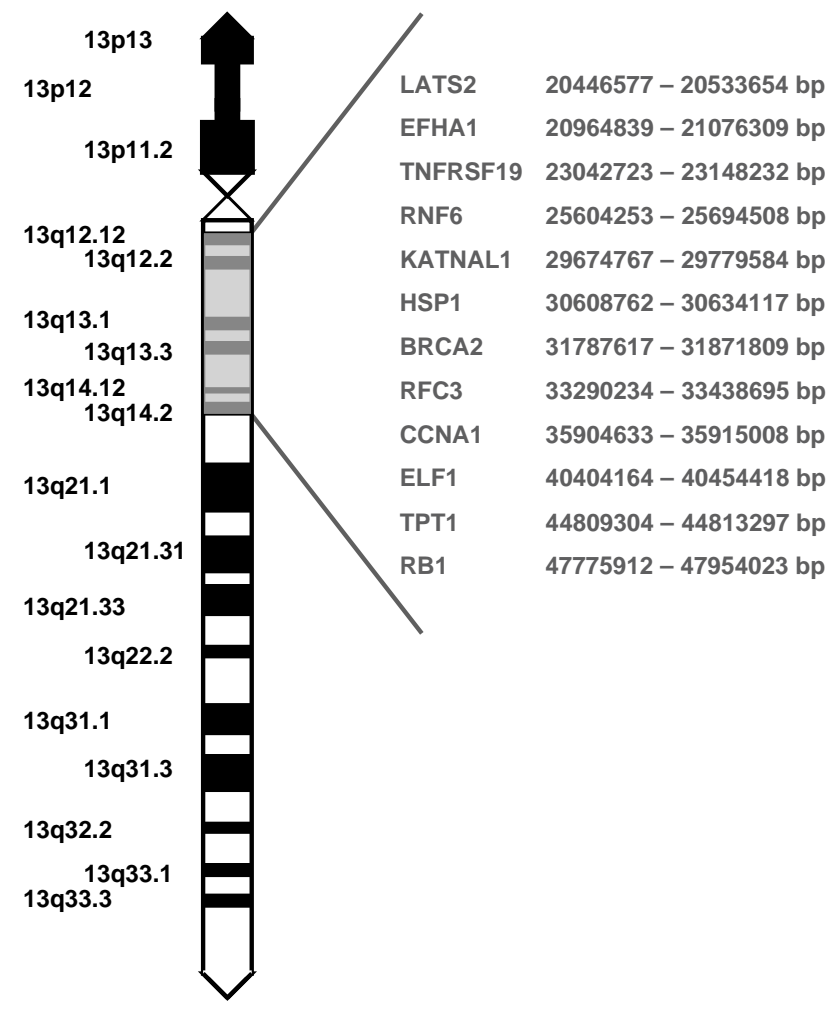

Fig. (1). Schematic of chromosome 13 showing the location of the twelve genes that were analyzed by qRT-PCR in patient-matched normal and tumor samples.

\section{Quantitative Reverse Transcription PCR (qRT-PCR) Gene Expression}

RT was conducted on all RNA samples using TaqMan RT Reagents (Applied Biosystems, Inc. (ABI), Foster City, CA, USA; Cat \# N808-0234), with random hexamers as the RT primers. cDNA was used immediately for all PCR reactions, with remaining cDNA stored at $-80^{\circ} \mathrm{C}$.

Fourteen commercially available optimized TaqMan primer/probe sets (Assays-on-Demand, ABI) were used in singleplex qPCR reactions. Beta-actin $(A C T B)$ has been shown to be the most stable endogenous control gene between esophageal tumor and normal tissue [23]: therefore, $A C T B$ was used as the endogenous control housekeeping gene for normalization. The assays for LATS2, EFHA1, TNFRSF19, RNF6, KATNAL1, HSP1, BRCA2, RFC3, $C C N A 1, E L F 1, T P T 1$, and $R B 1$ are cDNA specific. To assess reproducibility, two $B R C A 2$ primer/probes sets, each specific to a different exon of the gene (exon 4-5 and exon 13-14, respectively) were used in $\mathrm{qPCR}$ analysis of the cDNA samples, and a second qPCR analysis of BRCA2 exon 13-14 
Table 1. 13q Genes Evaluated by qRT-PCR Analysis of Microdissected ESCC Matched N and T Tissues

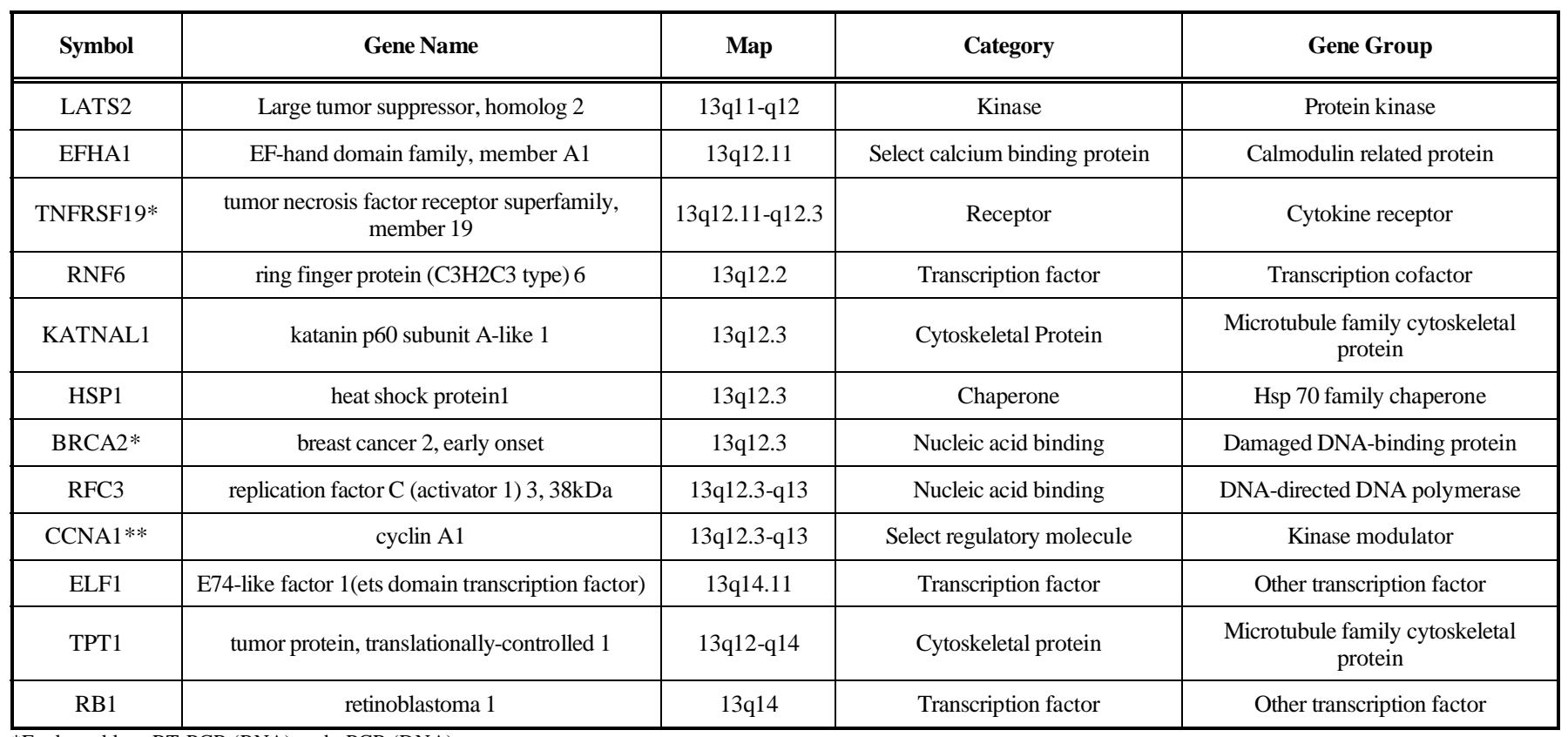

*Evaluated by qRT-PCR (RNA) and qPCR (DNA).

**Evaluated by qRT-PCR (RNA), qPCR (DNA), and IHC (protein).

was conducted using cDNA from a replicate LCM sample. Negligible contamination $(0.1 \%)$ of genomic DNA was confirmed by the observation of a cycle threshold $\left(\mathrm{C}_{\mathrm{T}}\right)$ of 10 comparing RT-negative with RT-positive samples. Triplicate qPCR assays were performed following RT. Controls consisting of total human esophagus RNA ( 12ng/ul; Ambion, Austin, TX, USA) were positive in all runs, and notemplate controls consisting of sterile molecular grade water were negative in all runs. Relative quantitation analysis of gene expression data was performed according to the $2^{-\Delta \Delta C T}$ method, with mean biologic fold change calculated directly from mean $\Delta \Delta \mathrm{C}_{\mathrm{T}}$ value [24].

\section{DNA Isolation, Quantitation, and Qualitation}

DNA extraction and isolation was conducted using the Qiagen DNeasy Blood \& Tissue Kit (Qiagen Inc., Cat \# 69504) according to manufacturer's protocol. DNA quantity and quality assessment was conducted using NanoDrop (NanoDrop Technologies) and Bioanalyzer (Agilent Technologies, Inc.) equipment, respectively, according to the manufacturer's protocol.

\section{Quantitative PCR (qPCR) DNA Measurement}

qPCR analysis was performed in triplicate singleplex reactions as previously described [19] using the extracted tumor and matched normal DNA samples. TaqMan primer/probe sets for genes of interest TNFSFR19, BRCA2 and CCNA1, and endogenous controls ACTB and CCR5 (C$\mathrm{C}$ chemokine receptor type 5) were employed in the qPCR reactions. All primer/probe sets were DNA specific and commercially available (Assays-on-Demand, ABI), except for CCR5, which was designed (CCR5 Probe 5'-Fam- CTG GGC TCA CTA TGC-MGB; CCR5 FP - TACCTGCTCAA CCTGGCCAT; CCR5 RP - TTCCAAAGTCCCACTGGGC). Controls consisting of human adult normal tissue esophagus DNA (BioChain Institute, Inc. Catalog \# D1234106) were positive in all runs, and no-template controls consisting of sterile molecular grade water were negative in all runs.

\section{Statistical Analysis of Gene Expression}

We tested for BRCA2 and CCNA1 up-regulation and TNFRSF19 and TPT1 down-regulation using a two-sided paired t-test and Bonferroni multiple correction. The mean difference in $\mathrm{CT}$ values by spatial location of the genes were also analyzed and plotted for LATS2, EFHA1, TNFRSF19, RNF6, KATNAL1, HSP1, average of 3 BRCA2 markers, RFC3, CCNA1, ELF1, TPT1, RB1. Log-base 10 P-values for two-sided t-tests comparing differences in the $C T$ values across successive genes (located next to each other) were analyzed with plots demonstrating the log p-value for a test of whether there is a shift between the ith and (i-1)th gene. A small p-value reflects evidence of a shift in mean values between the two markers. Bonferonni multiple comparisons correction was applied and statistical significance was considered a p-value less than $0.05 / 11=0.0045$ (accounting for 11 comparisons with a Bonferroni multiple comparisons correction).

\section{Immunohistochemistry (IHC)}

One case of formalin-fixed, paraffin-embedded tissue (FFPE) was immunohistochemically stained using biotinylated link streptavidin HRP (LSAB2, DAKO, K0675). A deparaffinized slide containing both normal epithelium and ESCC was heat-treated using a steamer (Black and Decker, HS2000) in Tris/EDTA buffer, PH9 (DAKO, S2367) for 20 minutes unmasking cyclin A epitopes. The slides were left in solution for addition 20 minutes to cool to room temperature and treated with peroxidase-blocking reagent (DAKO, S2001) to inhibit endogenous peroxidase activity. The sections were then incubated with a monoclonal anti-CCNA1 antibody (NCLcyclin $\mathrm{A}$, clone $6 \mathrm{E} 6,1: 50$ at room temperature). Afterwards, 
they were incubated with the biotinylated link antibody and streptavidin-peroxidase for 15 minutes each (DAKO, K0675). Subsequently, liquid DAB (diaminobenzidine) (DAKO, K3466) was applied for 5 minutes and the sections counterstained in hematoxylin (Lerner-2 Laboratories, Cat\# 1931413). Slides were then dehydrated and mounted with Cytoseal XYL (Richard-Allan, Cat\# 8312-4) mounting medium. Biotinylated link strepavidin HRP were prediluted visualization kits from DAKO, and anti-CCNA1 primary antibody was diluted with ready-to-use diluent (DAKO, S0809). CCNA1 expression in tissues was evaluated by IHC analysis using the DAKO Autostainer Universal Staining System (DAKO, S3800).

\section{RESULTS}

\section{Quantitative RT-PCR and qPCR Interrogation of Chromosome 13q12-14}

Transcript expression of 12 genes within the $13 \mathrm{q} \mathrm{LOH}$ region was analyzed by qRT-PCR using matched normal epithelium and squamous cell carcinoma from 17 cases (Fig. 1, Table 1). Full-thickness microdissection of the epithelium was performed for normal cell populations, and islands of invasive tumor were procured for the cancer samples. The RNA quantity was measured by NanoDrop (average tumor = $20.42 \mathrm{ng} / \mathrm{ul}$; average normal $=16.59 \mathrm{ng} / \mathrm{ul}$ ) and the RNA quality was determined by BioAnalyzer (average tumor $=$ 4.5 RIN; average normal $=5.1 \mathrm{RIN}$ ). For a normalization strategy, three steps were utilized: cell count during microdissection; total RNA measurement; and the use of a tissue specific endogenous housekeeping gene [19, 20].

Overall, five of the 12 transcripts were up-regulated in the majority of tumors (KATNAL1, HSP1, BRCA2, RFC3, CCNA1), two were down-regulated (TNFRSF19, TPT1), and five showed no consistent change (Table 2). The BRCA2 mRNA was increased an average of 5.02-fold in the ESCC samples and thus did not show evidence of loss-of function based on reduced transcript expression. CCNA1, a cell cycle regulatory gene, showed an increase of 129.36-fold, the most of any of the genes analyzed. The increases in $B R C A 2$ and $C C N A 1$ were both statistically significant $(\mathrm{P}<0.001)$.

The two down-regulated genes, TNFRSF19 and TPTI, showed a greater than 1.5 fold decrease in mRNA levels in 10 of 17 and 11 of 17 tumors, respectively (Table 2). Overall, TNFRS19 showed an average decrease in the tumors of 2.31 fold, and TPT1 had a mean decrease of 1.66. Although a trend toward decreased TNFRS19 and TPT1 transcript levels was observed in ESCC, these results were not statistically significant due in part to the relatively small number of cases analyzed.

Table 2. Individual Case $\Delta \Delta \mathbf{C}_{\mathrm{T}}$ Values of 13q Region Gene Expression

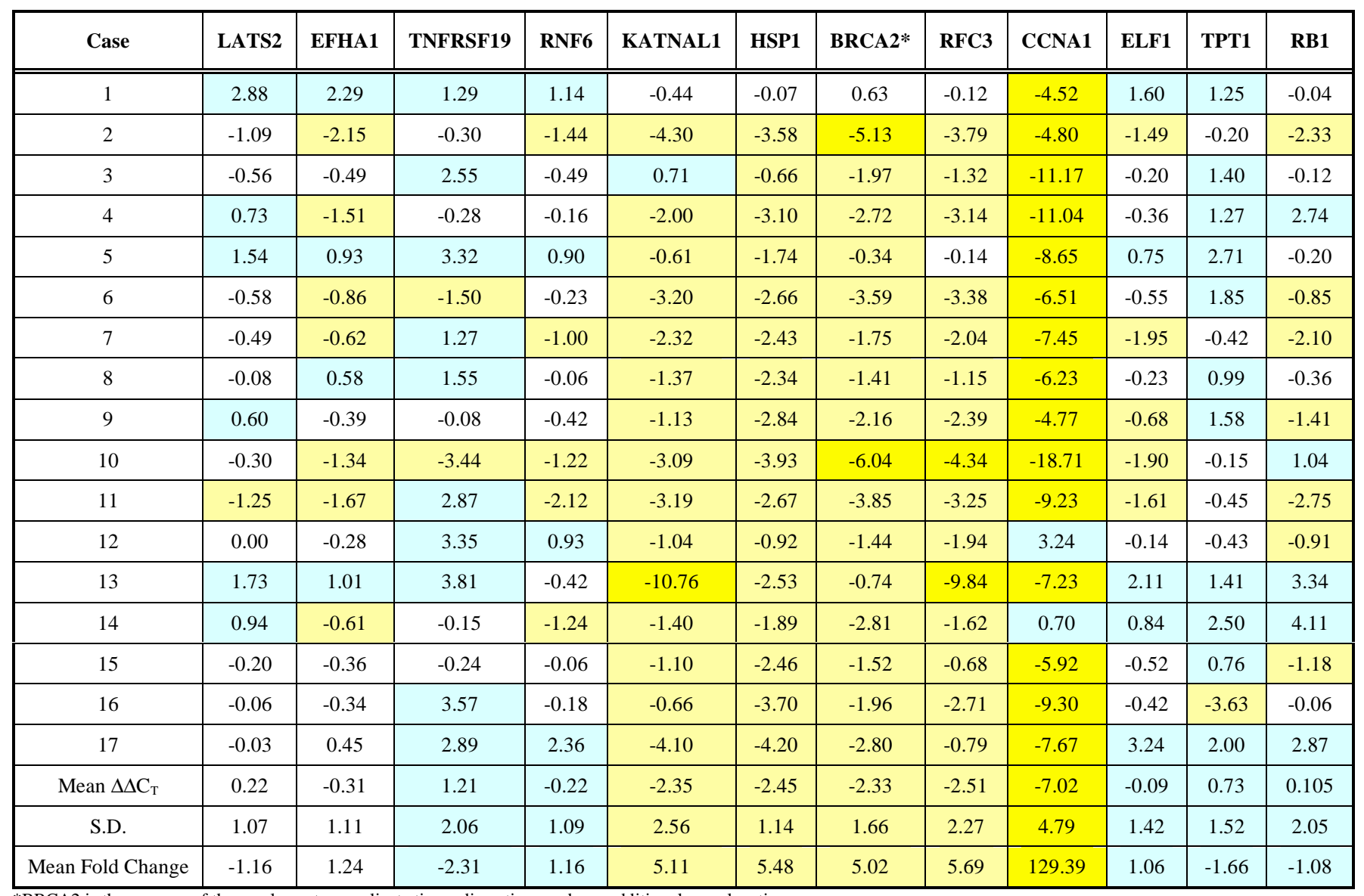

*BRCA2 is the average of three values: two replicate tissue dissections and one additional exon location. 
Table 3, Summary of 13q Gene Expression of Seventeen Cases of Microdissected Frozen ESCC Tissues and Matched Normal Tissues

\begin{tabular}{|c|c|c|c|c|c|c|c|}
\hline Gene & Map Value & Start (bp) & Stop (bp) & Size (bp) & $\operatorname{Mean} \Delta \Delta C_{T}$ & S.D. & Mean Fold Change* \\
\hline LATS2 & $13 q 11-q 12$ & 20446577 & 20533654 & 87077 & 0.22 & 1.07 & -1.16 \\
\hline EFHA1 & $13 \mathrm{q} 12.11$ & 20964839 & 21076309 & 111470 & -0.31 & 1.11 & 1.24 \\
\hline TNFRSFR $19^{\downarrow}$ & $13 q 12.11-q 12.3$ & 23042723 & 23148232 & 105509 & 1.21 & 2.06 & -2.31 \\
\hline RNF6 & $13 \mathrm{q} 12.2$ & 25604253 & 25694508 & 90255 & -0.22 & 1.09 & 1.16 \\
\hline KATNAL1 $^{\uparrow}$ & $13 q 12.3$ & 29674767 & 29779584 & 104817 & -2.35 & 2.56 & 5.11 \\
\hline $\mathrm{BRCA}^{\uparrow}{ }^{\uparrow}$ & $13 \mathrm{q} 12.3$ & 31787617 & 31871809 & 84192 & -2.33 & 1.66 & 5.02 \\
\hline $\mathrm{RFC}^{\uparrow}$ & $13 q 12.3-q 13$ & 33290234 & 33438695 & 148461 & -2.51 & 2.27 & 5.69 \\
\hline $\mathrm{CCNA}^{\uparrow}{ }^{\top *}$ & $13 q 12.3-q 13$ & 35904633 & 35915008 & 10375 & -7.02 & 4.79 & 129.39 \\
\hline ELF1 & $13 q 14.11$ & 40404164 & 40454418 & 50254 & -0.09 & 1.42 & 1.06 \\
\hline
\end{tabular}

Genes without arrow designation had no change in expression of tumor compared to normal ( $<1.5$ fold change).

${ }^{\downarrow}$ Down-regulation of gene expression in tumor tissue $(\mathrm{T}<\mathrm{N})$.

${ }^{\dagger}$ Up-regulation of gene expression in tumor tissue $(\mathrm{T}>\mathrm{N})$.

$\uparrow^{*} 4+(>20$ fold) Up-regulation of gene expression in tumor tissue $(\mathrm{T}>\mathrm{N})$.

* Mean fold change calculated from average $\Delta \Delta \mathrm{CT}$ value of all cases per gene.

The region spanning KATNALl to CCNAl on chromosome 13 demonstrated up-regulation of all five genes in the interval (Table 2) raising the possibility of a DNA amplicon that could involve $B R C A 2$ or known growth related genes such as CCNA1. Therefore, a statistical analysis was conducted using the qRT-PCR transcript expression data of the 12 genes in the region (Fig. 2). Significant mean shifts were observed between RNF6 and KATNAL1 (down, Pvalue $=4.19 \mathrm{E}-03$ ) and between $C C N A 1$ and ELF1 (up, Pvalue $=1.19 \mathrm{E}-06$ ), demarcating the region of consecutive gene up-regulation. Since the analysis of increased gene expression in this interval showed statistical significance, we measured DNA content at the TNFRS19, BRCA2, and CCNA1 genes using qPCR. No evidence of DNA amplification across the region of the five up-regulated genes was observed (data not shown).

\section{Immunohistochemical Analysis of CCNA1}

CCNA1 transcript was markedly elevated in ESCC and a commercial antibody for IHC was available, thus we qualitatively analyzed CCNA1 protein expression and distribution in normal and tumor cells. IHC analysis was conducted using formalin-fixed, paraffin-embedded tissue from one case in the study. The staining showed CCNA1 nuclear positivity in the basal region of normal epithelium where cell division occurs, but little to no expression in the more differentiated and superficial areas (Fig. 3). In contrast, both dysplastic epithelium and tumor showed CCNA1 staining throughout the lesions, consistent with cell division occurring widely in the transformed cell population. These results suggest that CCNA1 protein expression is associated with cell division in both normal and transformed cells.

\section{DISCUSSION}

The incidence of ESCC is notably high in several geographic regions of the world including Shanxi Province in China. At present, the etiology of this phenomenon is not known although both genetic and environment influences are suspected to play a role [1,2]. The BRCA2 gene on chromosome 13q12 may be involved in ESCC based on four lines of evidence: chromosome 13 is frequently deleted in this cancer type; there is a statistically significant difference in $\mathrm{LOH}$ on chromosome $13 \mathrm{q} 12$ between family history positive and family history negative cases; mutations in $B R C A 2$ are observed in a subset of tumors; and, germline mutations are present in affected kindreds [3-5, 13, 21, 22]. However, to date the exact role of the BRCA2 gene in ESCC is unclear as the frequency of tumor-specific and germline mutations is low, and the pattern does not fit the classic Knudsen two-hit model [5, 12-14].

To further investigate $B R C A 2$ and other genes on 13q1214, we examined expression levels in matched normal esophageal squamous epithelium and ESCC from seventeen patients using qRT-PCR. Overall, there were three major findings in the study. No evidence of loss of function of the $B R C A 2$ gene was observed based on reduced mRNA levels; CCNA1 was identified as a novel up-regulated gene in ESCC; and, the TNFRSF19 and TPT1 transcripts were reduced in the majority of tumors studied.

The increased levels of BRCA2 mRNA in ESCC likely represent a compensatory change in response to the extensive genomic instability that is observed in this tumor type. BRCA2 functions as a DNA repair enzyme via interaction with the RAD51 protein that is known to correct double-stranded DNA breaks and regulate homologous recombination during meiosis [25-29]. Loss-of-function of $B R C A 2$ is associated with several different tumor types with pronounced genomic instability. Further study of the role of $B R C A 2$ is warranted in order to both understand the role of the gene in ESCC and to evaluate its potential implications for treatment as inhibition of the compensatory cellular DNA repair response may be an effective therapeutic strategy by 

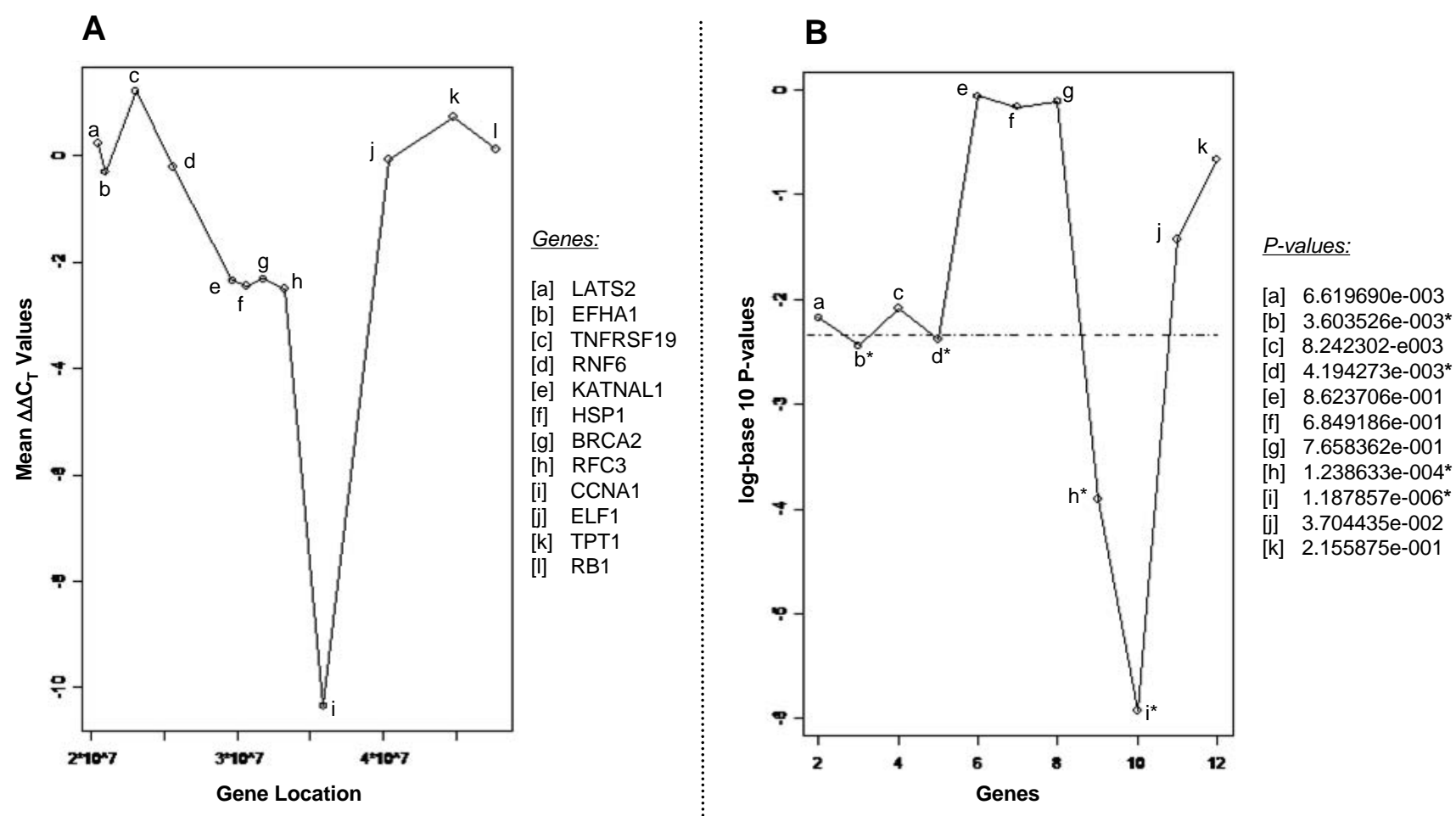

Fig. (2). Mean difference in CT values across each of the genes by spatial location. A. Plotted means. Genes analyzed: $a=$ LATS2, $b=$ EFHA1, $\mathrm{c}=$ TNFRSF19, $\mathrm{d}=\mathrm{RNF6}, \mathrm{e}=\mathrm{KATNAL1}, \mathrm{f}=\mathrm{HSP} 1, \mathrm{~g}=$ average of 3 BRCA 2 markers, $\mathrm{h}=\mathrm{RFC} 3, \mathrm{i}=\mathrm{CCNA} 1, \mathrm{j}=\mathrm{ELF} 1, \mathrm{k}=$ TPT1, 1 = RB1. B. Log-base 10 P-values for two-sided t-tests comparing differences in the CT values across successive genes (located next to each other). Dotted line reflects p-value $<0.05 / 11=0.0045$ (accounting for 11 comparisons with a Bonferonni multiple comparisons correction). $*=$ statistical significance representing a shift in mean structure.

making tumor cells susceptible to DNA damaging drugs [3034].

The CCNAl gene is located on chromosome 13q12.3-q13 and encodes a protein expressed in testis, brain and several leukemic cell lines that is thought to primarily function in the control of the meiotic cell cycle $[35,36]$. CCNA1 protein binds both $\mathrm{CDK} 2$ and $\mathrm{CDC} 2$ kinases, giving it two distinct kinase-related activities, one appearing in $\mathrm{S}$ phase, the other in $\mathrm{G} 2$, and thus regulating separate functions of the cell cycle [37]. CCNA1 also binds to important cell cycle regulators such as $\mathrm{Rb}$ family proteins transcription factor E2F-1 and the p21 protein family, and appears to be a downstream player in p53-dependent apoptosis and G2 arrest [38-40]. In cancer, CCNA1 can induce G2 cell cycle arrest, polyploidy, apoptosis, and mitotic catastrophe as has been reported for non-small cell lung, ovarian, and renal carcinoma cells [39]. Two primary renal cell carcinomas expressing mutated p53 exhibited reduced or absent expression of CCNA1 relative to the corresponding normal tissue [39]. In prostate cancer, CCNA1 mediates VEGF expression in cooperation with Rband androgen-dependent pathways [38]. IHC expression of CCNA1 differs between BPH and cancer as BPH staining is cytoplasmic and pale whereas in cancer the staining is nuclear and related to histologic grade with aggressive tumors showing more intense staining.

In ESCC, we showed that CCNAI mRNA was elevated in tumors suggesting the gene may be important in the etiology of this neoplasm. While this still may be true, the use of tissue immunostaining as a 'biological filter' indicates that the CCNA1 protein appears to be associated with both normal and pathological cell growth and thus may not be a tumor specific change per se. In other words, the apparent up-regulation of CCNAl mRNA in ESCC could be due, at least in part, to the restricted expression in normal epithelium (basal layer only) versus the diffuse expression seen in tumors. Since we microdissected the full thickness of the normal esophageal epithelium, including the differentiated layers without dividing cells, the expression measurements of growth-related genes were biased in favor of the tumor samples. These results highlight that an important next step in identifying and/or validating true growth-related, tumor specific gene expression changes will require direct comparison of microdissected basal esophageal cells (normal growth) versus ESCC (pathological growth) using expression arrays or qRT-PCR analyses [41].

Both the TNFRS19 and TPT1 transcripts were downregulated in the majority of tumors studied, thus further analysis of these two genes in additional cases of ESCC at both the genomic and protein levels are indicated. The TNFRS19 gene is located on chromosome 13q12.11-12.3 and is a member of the tumor necrosis receptor superfamily $[42,43]$. The protein functions as a cytokine receptor involved with caspase-independent apoptosis and is highly expressed during embryonic development [44]. Receptor ligands are thought to include members of the TRAF family, genes that are important in Drosophila developmental processes via interactions with the JNK and NF-kappaB signaling pathways $[45,46]$. Based on its cellular functions and known interactions with tumor necrosis factors, loss of 

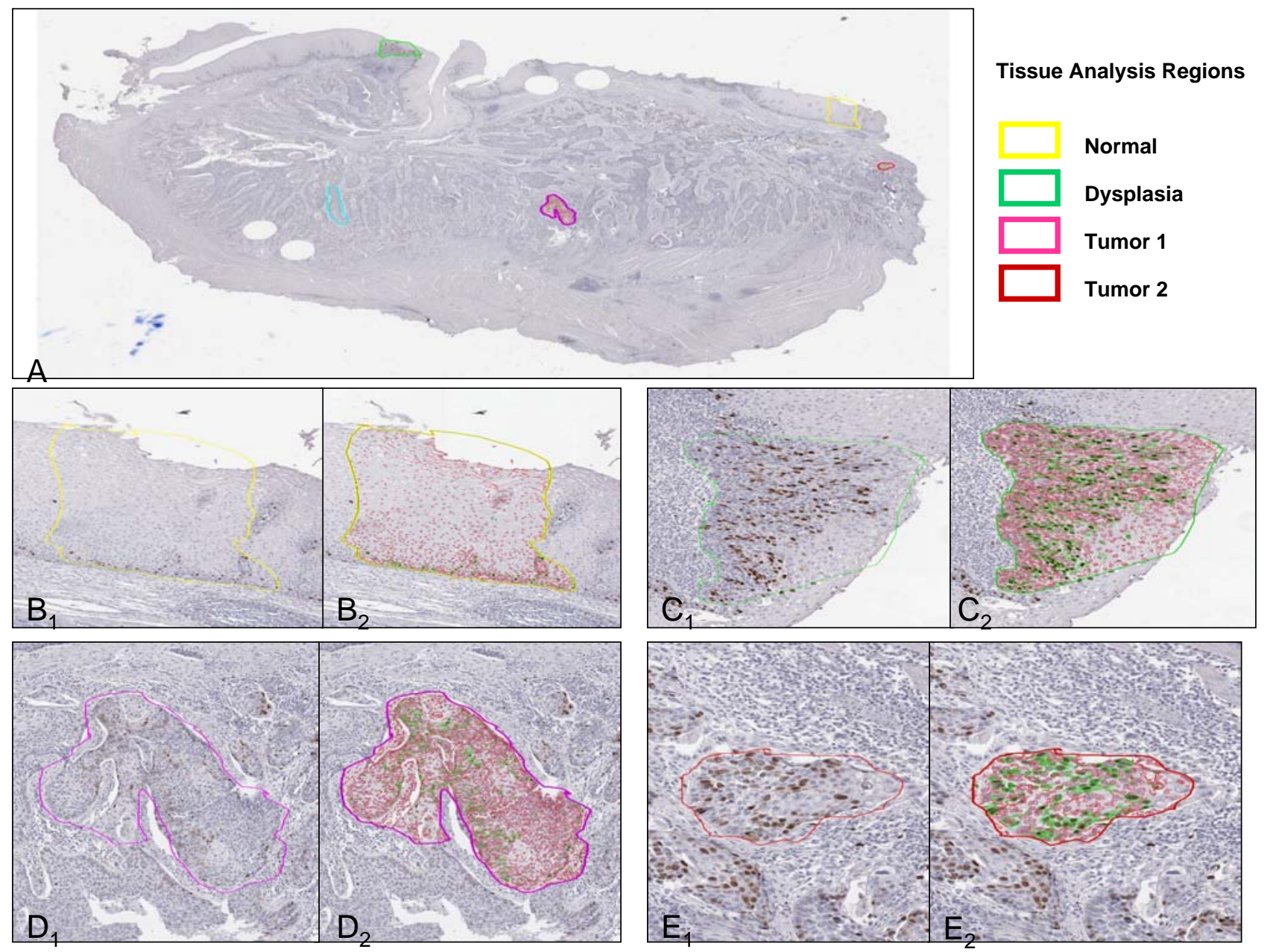

Fig. (3). CCNA1 Immunnohistochemistry (IHC). A. Histological map with image analysis areas outlined. B. Normal epithelium. C. Zone of dysplasia. D. Region 1 of tumor. E. Region 2 of tumor. ${ }_{1}=$ Area to be analyzed; ${ }_{2}=$ Image analysis annotation, green nuclei $=$ positive, pink nuclei $=$ negative. IHC stained, original magnification, x20 (A), x200 (B-E).

TNFRS19 in ESCC is a plausible tumor suppressor gene candidate. The TPT1 gene is located on 13q12-14 and its protein product has been reported to be involved in multiple different cellular functions including those related to cellular growth and differentiation [47-53]. At present, the potential role of TPT1 in ESCC formation and development is not clear; however further investigation in more cases is warranted given its chromosomal location and reduced expression in tumors.

\section{CONCLUSION}

Quantitative transcript analysis of patient matched normal and tumor samples indicate that the BRCA2 gene does not exhibit loss-of-function in ESCC based on reduced mRNA levels. Additional study in a larger patient population will be required to understand the potential role of the CCNA1, TNFRS19, and TPT1 genes in this cancer type.

\section{ACKNOWLEDGEMENTS}

We thank Wusheng Yan and Liang Zhu for discussions about this study, Jeffery Hanson for providing CCR5 primers and probe set sequences, and Keith Killian for DNA extraction advice.
The research was supported by the Intramural Research Programs of the Center for Cancer Research, and the Division of Cancer Epidemiology and Genetics, National Cancer Institute, NIH.

\section{CONFLICT OF INTEREST}

The authors confirm that this article content has no conflicts of interest.

\section{REFERENCES}

[1] Hu N, Dawsey SM, Wu M, et al. Family history of oesophageal cancer in Shanxi Province, China. Eur J Cancer 1991; 27: 1336.

[2] $\mathrm{Hu} \mathrm{N}$, Dawsey SM, Wu M, et al. Familial aggregation of oesophageal cancer in Yangcheng County, Shanxi Province, China. Int J Epidemiol 1992; 21: 877-82.

[3] Roth MJ, Hu N, Emmert-Buck MR, et al. Genetic progression and heterogeneity associated with the development of esophageal squamous cell carcinoma. Cancer Res 2001; 61: 4098-104.

[4] Lo HS, Hu N, Gere S, et al. Identification of somatic mutations of the RNF6 gene in human esophageal squamous cell carcinoma. Cancer Res 2002; 62: 4191-3.

[5] $\mathrm{Hu} \mathrm{N}$, Goldstein AM, Albert PS, et al. Evidence for a familial esophageal cancer susceptibility gene on chromosome 13 . Cancer Epidemiol Biomarkers Prev 2003; 12: 1112-5.

[6] Li G, Hu N, Goldstein AM, et al. Allelic loss on chromosome bands 13q11-q13 in esophageal squamous cell carcinoma. Genes Chromosomes Cancer 2001; 31: 390-7. 
[7] Li WJ, Hu N, Su H, et al. Allelic loss on chromosome 13q14 and mutation in deleted in cancer 1 gene in esophageal squamous cell carcinoma. Oncogene 2003; 22: 314-8.

[8] $\mathrm{Hu} \mathrm{N}, \mathrm{Su} \mathrm{H}, \mathrm{Li}$ WJ, et al. Allelotyping of esophageal squamouscell carcinoma on chromosome 13 defines deletions related to family history. Genes Chromosomes Cancer 2005; 44: 271-8.

[9] Hu N, Wang C, Hu Y, et al. Genome-wide association study in esophageal cancer using GeneChip mapping 10K array. Cancer Res 2005; 65: 2542-6.

[10] Hu N, Wang C, Hu Y, et al. Genome-wide loss of heterozygosity and copy number alteration in esophageal squamous cell carcinoma using the Affymetrix GeneChip Mapping $10 \mathrm{~K}$ array. BMC Genomics 2006; 7: 299.

[11] $\mathrm{Hu} \mathrm{N}$, Wang $\mathrm{C}, \mathrm{Ng} \mathrm{D}$, et al. Genomic characterization of esophageal squamous cell carcinoma from a high-risk population in China. Cancer Res 2009; 69: 5908-17.

[12] $\mathrm{Hu} \mathrm{N}, \mathrm{Li} \mathrm{G}, \mathrm{Li}$ WJ, et al. Infrequent mutation in the BRCA2 gene in esophageal squamous cell carcinoma. Clin Cancer Res 2002; 8: 1121-6.

[13] Hu N, Li WJ, Su H, et al. Common genetic variants of TP53 and BRCA2 in esophageal cancer patients and healthy individuals from low and high risk areas of northern China. Cancer Detect Prev 2003; 27: 132-8

[14] Hu N, Wang C, Han XY, et al. Evaluation of BRCA2 in the genetic susceptibility of familial esophageal cancer. Oncogene 2004; 23: 852-8.

[15] Kaushal M, Chattopadhyay I, Phukan R, et al. Contribution of germ line BRCA2 sequence alterations to risk of familial esophageal cancer in a high-risk area of India. Dis Esophagus 2009; 23: 71-5.

[16] Akbari MR, Malekzadeh R, Nasrollahzadeh D, et al. Germline BRCA2 mutations and the risk of esophageal squamous cell carcinoma. Oncogene 2008; 27: 1290-296.

[17] Gardner, LB. Nonsense-mediated RNA decay regulation by cellular stress: Implications for tumorigenesis. Mol Cancer Res 2010; 8: 295-308.

[18] Erickson HS, Gillespie JW, Emmert-Buck MR. Tissue microdissection. Methods Mol Biol 2008; 424: 433-48.

[19] Erickson HS, Albert PS, Gillespie JW, et al. Quantitative RT-PCR gene expression analysis of laser microdissected tissue samples. Nat Protoc 2009; 4: 902-22.

[20] Erickson HS, Albert PS, Gillespie JW, et al. Assessment of normalization strategies for quantitative RT-PCR using microdissected tissue samples. Lab Invest 2007; 87: 951-62.

[21] Hu N, Roth MJ, Emmert-Buck MR, et al. Allelic loss in esophageal squamous cell carcinoma patients with and without family history of upper gastrointestinal tract cancer. Clin Cancer Res 1999; 5: 3476-82.

[22] $\mathrm{Hu} \mathrm{N}$, Roth MJ, Polymeropolous M, et al. Identification of novel regions of allelic loss from a genomewide scan of esophageal squamous-cell carcinoma in a high-risk Chinese population. Genes Chromosomes Cancer 2000; 27: 217-28.

[23] Jin P, Zhao Y, Ngalame Y, Panelli MC, et al. Selection and validation of endogenous reference genes using a high throughput approach. BMC Genomics 2004; 5: 55.

[24] Livak KJ, Schmittgen TD. Analysis of relative gene expression data using real-time quantitative PCR and the 2(-Delta Delta C(T)) Method. Methods 2001; 25: 402-8.

[25] Sharan SK, Morimatsu M, Albrecht U, et al. Embryonic lethality and radiation hypersensitivity mediated by Rad51 in mice lacking BRCA2. Nature 1997; 386: 804-10.

[26] Patel KJ, Yu VP, Lee H, et al. Involvement of BRCA2 in DNA repair. Mol Cell 1998; 1: 347-57.

[27] Cheung AM, Hande MP, Jalali F, et al. Loss of BRCA2 and p53 synergistically promotes genomic instability and deregulation of Tcell apoptosis. Cancer Res 2002; 62: 6194-204.

[28] Lee H. Impaired phosphorylation and mis-localization of Bub1 and BubR1 are responsible for the defective mitotic checkpoint function in BRCA2-mutant thymic lymphomas. Exp Mol Med 2003; 35: 448-53.
[29] Atanassov BS, Barrett JC, Davis BJ. Homozygous germ line mutation in exon 27 of murine Brca2 disrupts the Fancd2-BRCA2 pathway in the homologous recombination-mediated DNA interstrand cross-links' repair but does not affect meiosis. Genes Chromosomes Cancer 2005; 44: 429-37.

[30] Nelson NJ. BRCA2 research is on a fast track to the clinic. J Natl Cancer Inst 1998; 90: 1502-4.

[31] Bennett LM, McAllister KA, Malphurs J, et al. Mice heterozygous for a BRCA1 or BRCA2 mutation display distinct mammary gland and ovarian phenotypes in response to diethylstilbestrol. Cancer Res 2000; 60: 3461-9.

[32] Foulkes WD. BRCA1 and BRCA2: chemosensitivity, treatment outcomes and prognosis. Fam Cancer 2006; 5: 135-42.

[33] Edwards SL, Brough R, Lord CJ, et al. Resistance to therapy caused by intragenic deletion in BRCA2. Nature 2008; 451: 11115.

[34] Cavelier C, Didier C, Prade N, et al. Constitutive activation of the DNA damage signaling pathway in acute myeloid leukemia with complex karyotype: potential importance for checkpoint targeting therapy. Cancer Res 2009; 69: 8652-61.

[35] Schrader M, Ravnik S, Muller-Tidow C, et al. Quantification of cyclin A1 and glyceraldehyde-3-phosphate dehydrogenase expression in testicular biopsies of infertile patients by fluorescence real-time RT-PCR. Int J Androl 2002; 25: 202-9.

[36] Diederichs S, Baumer N, Ji P, et al. Identification of interaction partners and substrates of the cyclin A1-CDK2 complex. J Biol Chem 2004; 279: 33727-41.

[37] Muller-Tidow C, Ji P, Diederichs S, et al. The cyclin A1-CDK2 complex regulates DNA double-strand break repair. Mol Cell Biol 2004; 24: 8917-28

[38] Wegiel B, Bjartell A, Ekberg J, et al. A role for cyclin A1 in mediating the autocrine expression of vascular endothelial growth factor in prostate cancer. Oncogene 2005; 24: 6385-93.

[39] Rivera A, Mavila A, Bayless KJ, et al. Cyclin A1 is a p53-induced gene that mediates apoptosis, G2/M arrest, and mitotic catastrophe in renal, ovarian, and lung carcinoma cells. Cell Mol Life Sci 2006; 63: 1425-39.

[40] Nakamaki T, Hamano Y, Hisatake J, et al. Elevated levels of cyclin A1 and A (A2) mRNA in acute myeloid leukaemia are associated with increased survival. Br J Haematol 2003; 123: 72-80.

[41] Cole KA, Krizman DB, Emmert-Buck MR. The genetics of cancer-a 3D model. Nat Genet 1999; 21: 38-41.

[42] Robertson NG, Khetarpal U, Gutierrez-Espeleta GA, et al. Isolation of novel and known genes from a human fetal cochlear cDNA library using subtractive hybridization and differential screening. Genomics 1994; 23: 42-50.

[43] Spanjaard RA, Whren KM, Graves C, et al. Tumor necrosis factor receptor superfamily member TROY is a novel melanoma biomarker and potential therapeutic target. Int J Cancer 2007; 120: 1304-10.

[44] Kojima T, Morikawa Y, Copeland NG, et al. TROY, a newly identified member of the tumor necrosis factor receptor superfamily, exhibits a homology with Edar and is expressed in embryonic skin and hair follicles. J Biol Chem 2000; 275: 20742-7.

[45] Eby MT, Jasmin A, Kumar A, et al. TAJ, a novel member of the tumor necrosis factor receptor family, activates the c-Jun Nterminal kinase pathway and mediates caspase-independent cell death. J Biol Chem 2000; 275: 15336-42.

[46] Naito A, Yoshida H, Nishioka E, et al. TRAF6-deficient mice display hypohidrotic ectodermal dysplasia. Proc Natl Acad Sci USA 2002; 99: 8766-71.

[47] Gross B, Gaestel M, Bohm H, et al. cDNA sequence coding for a translationally controlled human tumor protein. Nucleic Acids Res 1989; 17: 8367.

[48] MacDonald SM, Paznekas WA, Jabs EW. Chromosomal localization of tumor protein, translationally-controlled 1 (TPT1) encoding the human histamine releasing factor (HRF) to 13q12->q14. Cytogenet Cell Genet 1999; 84: 128-9.

[49] MacDonald SM, Rafnar T, Langdon J, et al. Molecular identification of an IgE-dependent histamine-releasing factor. Science 1995; 269: 688-90. 
[50] Li F, Zhang D, Fujise K. Characterization of fortilin, a novel antiapoptotic protein. J Biol Chem 2001; 276: 47542-9.

[51] Bommer UA, Borovjagin AV, Greagg MA, et al. The mRNA of the translationally controlled tumor protein P23/TCTP is a highly structured RNA, which activates the dsRNA-dependent protein kinase PKR. RNA 2002; 8: 478-96.
$[52]$

Tuynder M, Susini L, Prieur S, et al. Biological models and genes of tumor reversion: cellular reprogramming through tpt1/TCTP and SIAH-1. Proc Natl Acad Sci USA 2002; 99: 14976-81.

[53] Yarm FR. Plk phosphorylation regulates the microtubulestabilizing protein TCTP. Mol Cell Biol 2002; 22: 6209-21.

(c) Erickson et al.; Licensee Bentham Open.

This is an open access article licensed under the terms of the Creative Commons Attribution Non-Commercial License (http://creativecommons.org/licenses/by$\mathrm{nc} / 3.0 /$ ) which permits unrestricted, non-commercial use, distribution and reproduction in any medium, provided the work is properly cited. 